\title{
Kinetic Alfvén Eigenmodes at ASDEX Upgrade
}

\author{
Ph. Lauber ${ }^{1}$, M. Brüdgam ${ }^{1}$, D. Curran ${ }^{2}$, V. Igochine ${ }^{1}$, \\ K. Sassenberg ${ }^{2}$, S. Günter ${ }^{1}$, M. Maraschek ${ }^{1}$, \\ M. García-Muñoz ${ }^{1}$, N. Hicks ${ }^{1}$ and the ASDEX Upgrade Team \\ ${ }^{1}$ Max-Planck-Institut für Plasmaphysik, EURATOM-Association, Garching, \\ Germany \\ ${ }^{2}$ Department of Physics, University College Cork, EURATOM-Association DCU, \\ Cork, Ireland \\ E-mail: pwl@ipp.mpg.de
}

\begin{abstract}
On the way to a comprehensive understanding of the properties of a burning plasma the physics of super-thermal particles due to external heating and fusion reactions plays a key role. Especially Alfvén and Alfvén-acoustic type instabilities are predicted to strongly interact with the fast particle population and to contribute critically to the radial redistribution of the energetic ions.

This paper focuses on the comparison of the kinetic dispersion relation for BAEs/GAMs [1] with numerical results obtained by the gyrokinetic eigenvalue code LIGKA [2] and experimental findings at ASDEX Upgrade. It is shown that thermal ions with a finite perpendicular energy (circulating and trapped) modify the dispersion relation significantly for low frequencies. The resulting frequency down-shift together with shaping and diamagnetic effects is crucial to explain the mode frequency as measured at ASDEX Upgrade stressing the importance of a kinetic description for frequencies comparable to the thermal ion transit frequency.

In the second part the BAE frequency behaviour during a sawtooth-cycle is investigated and the possibility of an accurate q-profile determination via kinetic Alfvén spectroscopy is discussed.
\end{abstract}

PACS numbers: 52.55.Fa, 52.65.Tt, 52.35.Bj 


\section{Introduction}

Although beta-induced Alfvén eigenmodes (BAEs) [3, 4] and geodesic acoustic modes (GAMs) [5] are closely related and are in fact in the long wave-length limit described by the same dispersion relation $[6,7,8,9,10]$, their experimental manifestation and their consequences for tokamak research are quite different. Whereas BAEs are electromagnetic, $n \neq 0$ perturbations, driven by energetic particles, usually located in the plasma core at roughly $30 \%$ to $50 \%$ of the toroidal Alfvén eigenmode (TAE) frequency, the GAMs are $n=0$, mostly electrostatic modes closely related to the zonal flow and turbulence physics especially at the plasma edge.

Their kinetic dispersion relation has been first derived by Zonca [1] in the ballooning representation. A generalised expression including finite Larmor orbit and finite orbit width effects can be found in reference [11]. Recently, several alternative derivations (e.g. by Nguyen via Fourier expansion, high-q limit [9] and by Elfimov via dielectric tensor formulation [12]) were reported. Furthermore, also elongation effects for the GAM dispersion relation were analytically and numerically investigated $[13,14]$.

The relevant dispersion relation was re-derived [15] for the gyrokinetic model [16] underlying the eigenvalue code LIGKA [2]. This derivation is also based on a Fourier expansion in the poloidal angle but keeps the full resonances, i.e. is valid for low $q$. Keeping the $m \pm 1$-sidebands, retaining the geodesic curvature and the sound wave coupling by an appropriate approximation of the propagator integrals, leads to:

$$
\begin{aligned}
& \omega^{2}\left(1-\frac{\omega_{* p}}{\omega}\right)-k_{\|}^{2} \omega_{A}^{2} R_{0}^{2}=2 \frac{v_{t h i}^{2}}{R_{0}^{2}}\left(-\left[H\left(x_{m-1}\right)+H\left(x_{m+1}\right)\right]+\right. \\
& \left.\tau\left[\frac{N^{m}\left(x_{m-1}\right) N^{m-1}\left(x_{m-1}\right)}{D^{m-1}\left(x_{m-1}\right)}+\frac{N^{m}\left(x_{m+1}\right) N^{m+1}\left(x_{m+1}\right)}{D^{m+1}\left(x_{m+1}\right)}\right]\right)
\end{aligned}
$$

with $x_{m}=\frac{\omega}{k_{\|, m} v_{t h}}, v_{t h i}^{2}=2 T_{i} / m_{i}, \omega_{p *}=\omega_{* n}+\omega_{* T}=T_{i} /(e B) k_{\theta}(\nabla n / n)(1+\eta)$ with $\eta=\frac{\nabla T}{T} / \frac{\nabla n}{n}, D\left(x_{m}\right)=\left[1+\tilde{D}\left(x_{e, m}\right)\right]+\tau\left[1+\tilde{D}\left(x_{i, m}\right)\right], N^{m}\left(x_{m}\right)=\tilde{N}^{m}\left(x_{i, m}\right)-\tilde{N}^{m}\left(x_{e, m}\right)$, $\tilde{D}(x)=\left(1-\frac{\omega_{*}}{\omega}\right) x Z(x)-\frac{\omega_{*}}{\omega} \eta\left(x^{2}+x Z(x)\left(x^{2}-\frac{1}{2}\right)\right), 2 \tilde{N}^{m}(x)=\left(1-\frac{\omega_{*}^{m}}{\omega}\right)\left[x^{2}+\right.$ $\left.\left.x Z(x)\left(x^{2}+\frac{1}{2}\right)\right]-\frac{\omega_{*}^{m}}{\omega} \eta\left[x^{4}+\frac{x^{2}}{2}\right)+x Z(x)\left(\frac{1}{4}+x^{4}\right)\right], H\left(x_{m}\right)=\tilde{H}\left(x_{m, i}\right)+\tau \tilde{H}\left(x_{m, e}\right)$, $\tilde{H}\left(x_{m}\right)=\frac{1}{2}\left[\left(1-\frac{\omega^{*}}{\omega}\right) \tilde{F}\left(x_{m}\right)-\eta \frac{\omega^{*}}{\omega} \tilde{G}\left(x_{m}\right)\right], 2 \tilde{F}(x)=x Z(x)\left(\frac{1}{2}+x^{2}+x^{4}\right)+\frac{3 x^{2}}{2}+x^{4}$, $2 \tilde{G}(x)=x Z(x)\left(\frac{3}{4}+x^{2}+\frac{x^{4}}{2}+x^{6}\right)+2 x^{2}+x^{4}+x^{6}$ and $Z(x)$ the plasma dispersion function. Although obtained in a completely different way, eqn. (1) is very similar (same coefficients) to the ballooning formulation result. The asymmetry in the $\omega_{*}$ terms (omitted in the first derivation based on LIGKA's set of equation in [15]) was pointed out by Zonca $[17,18]$.

The aim of this paper is the following: after describing the experimental parameters, the validity of treating only circulating thermal ions with vanishing perpendicular energy, i.e. $\Lambda=\mu B_{0} / E=0$, is investigated. All analytical models except a very recent work including deeply trapped particles [19] rely on this simplification. Due to the one-to-one correspondence of the analytical and the numerical coefficients in a linear eigenvalue code, the correctness of the numerics and the limits of the analytical approximations 
can be checked.

Therefore, this study assesses also the question if a fully kinetic treatment of the thermal background species for this low- frequency regime is important or if MHD/fluid theories are sufficient. It will be shown, that diamagnetic and shaping effects together with $\Lambda \neq 0$ and trapped particle contributions modify the dispersion relation in a non-trivial way. The resulting downshift of the BAE/GAM continuum frequency is essential to explain the experimentally measured BAE frequency. In the second part the evolution of the BAE continuum accumulation point during a sawtooth cycle at ASDEX Upgrade is followed analytically and is compared to the experimental findings. This procedure assumes that the mode is mainly a gap mode, or at least close to the gap. In the experiment no bursty mode behavior can be seen. We take this as an indication that the mode drive is too weak to excite the energetic-particle branch and therefore the gapmode hypothesis seems to be justified. Moreover, this analysis checks the hypothesis that the mode described in ref. [20] ('sierpes') is a BAE mode.

In the last part the simultaneous appearance of Alfvén-Cascades (RSAEs) and BAEs is investigated, relying on radial localisation information given by the soft-X-ray diagnostic $[21]$.

\section{Experimental values}

\#20488 is a low density discharge with $800 k A$ and an ICRF heating power of $4.5 \mathrm{MW}$. The observed sawtooth inversion radius derived from ECE measurements is $\varrho_{\text {pol }}=0.37$ or $r=18 \mathrm{~cm}$, determining an upper bound for the $q=1$ surface. From the profiles shown in fig. 1 (fit of several combined density diagnostics and ECE data) the gradients are found to be $\nabla n / n=-1.9 \mathrm{~m}^{-1}$ and $\nabla T_{e} / T_{e}=-4.5 \mathrm{~m}^{-1}$ before the sawtooth crash and $\nabla T_{e} / T_{e}=-1.8 m^{-1}$ after the crash. The density profile is assumed to stay constant during the sawtooth cycle, in agreement with experimental observations. With $k_{\theta}=-m / r_{s}=m / 18 \mathrm{~cm}=m \cdot 5.5 \mathrm{~m}^{-1}, T_{e}=3.5 \mathrm{keV}$ and $B=2.43 T$ one obtains $f_{* p}=\omega_{* p} / 2 \pi=\left(\omega_{* n}+\omega_{* T}\right) / 2 \pi=m(2.3 \mathrm{kHz}+5.5 \mathrm{kHz})=m \cdot 7.8 \mathrm{kHz}$ for the steep profile and $f_{* p}=\omega_{* p} / 2 \pi=m(2.3 \mathrm{kHz}+2.2 \mathrm{kHz})=m \cdot 4.5 \mathrm{kHz}$ for the flat profile. As error one can assume roughly $10-15 \%$ for the temperature gradient and about $20 \%$ for the density gradient. For a typical mode number of $n=5, m=5$ the diamagnetic frequency can be up to a third of the observed BAE frequency and thus significantly modifies its' dispersion relation. It should be noted that these gradients do not exceed the typical values determined in transport studies at ASDEX Upgrade where the critical gradient length limited by the onset of strong electron heat transport was found to be above $\nabla T_{e} / T_{e}=-6 m^{-1}[22]$.

The thermal velocity of the deuterium ions at the $q=1$ surface is $0.095 v_{A 0}(\beta \approx 1.8 \%)$ with $v_{A 0}=B_{0} / \sqrt{\mu_{0} n_{i 0} m_{i}}=6.0 \cdot 10^{6} \mathrm{~m} / \mathrm{s} . \quad R_{0}$ is $1.72 \mathrm{~m}$, the mid-plane minor radius $a=0.5 \mathrm{~m}$. For this discharge, no ion temperature measurements are available, therefore $\tau=T_{e} / T_{i}=1$ is assumed. This assupmtion is motivated by previous studies of 

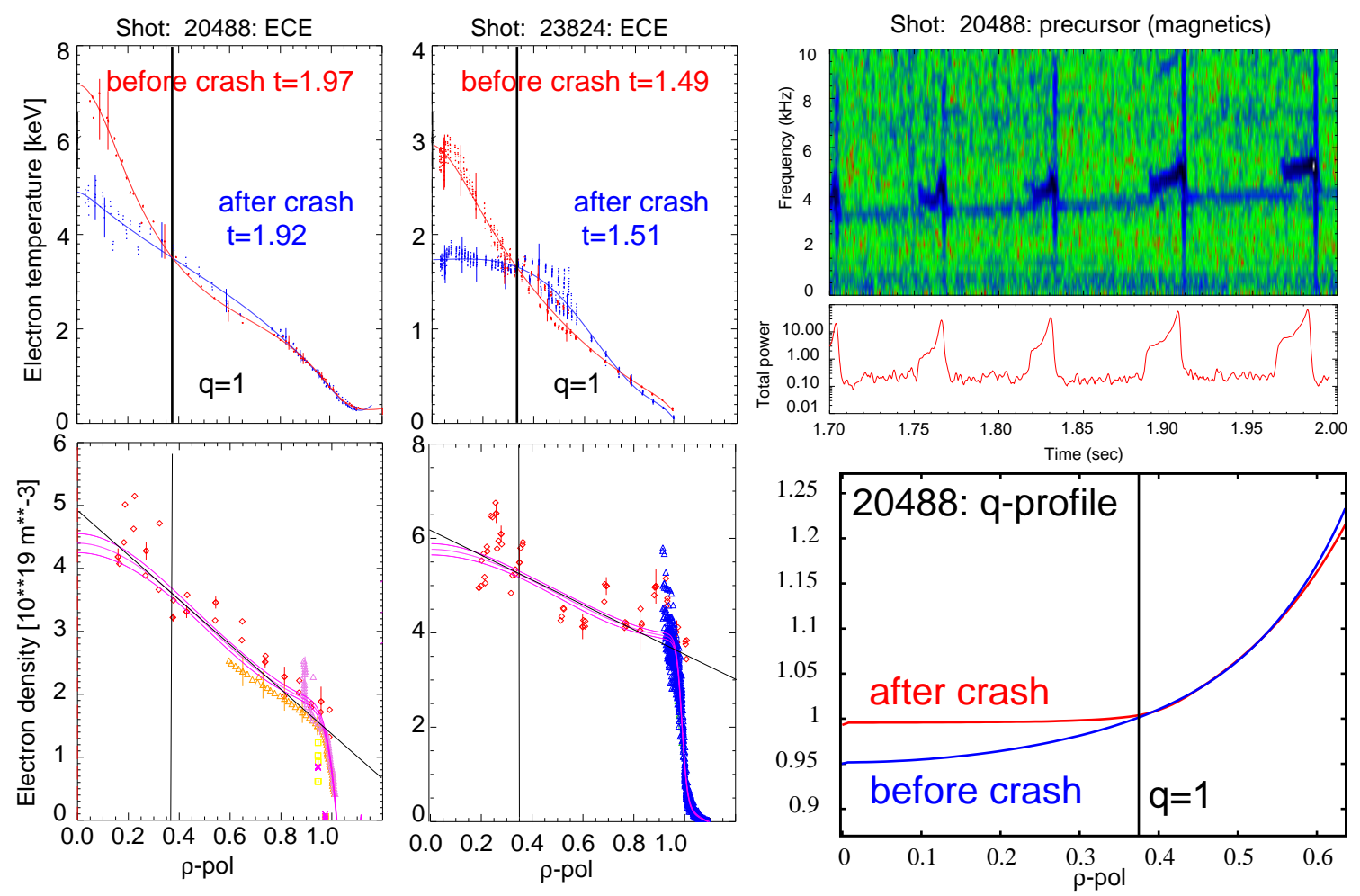

Figure 1. Density (as obtained by a combination of core Thomson scattering data, interferometer, reflectometer and Lithium beam data), temperature (ECE) and qprofiles for the two discharges discussed in this paper. Sawtooth precursors with a frequency of $4 \mathrm{kHz}$ at \#20488 are seen in the magnetics.

IRCF heated discharges at ASDEX Upgrade[23].In shot \#20488 a sawtooth precursor of $4 \mathrm{kHz}$ was observed, determining the approximate toroidal rotation frequency at the $q=1$ surface.

\section{Comparison of analytical and numerical results}

With the parameters above the dispersion relation (1) is investigated and compared to numerical results obtained by the LIGKA/HAGIS [24] codes. As a first point, the approximation for the crucial operator containing the geodesic curvature $\left(v_{d} \cdot \nabla\right)_{r}=$ $\omega_{d}^{r} \sin \theta \approx \frac{v_{t h i}^{2}}{\Omega_{c i} R_{0}} \sin \theta \frac{\partial}{\partial r}$ is investigated. Fig. 2 shows how for increasing perpendicular energy i.e. increasing $\Lambda=\mu B_{0} / E$ the radial drifts start to deviate from the fast circulating particle approximation $\theta \approx \omega_{t} \cdot t$ where $\omega_{t}$ is the transit frequency, $t$ the time variable and $\theta$ the poloidal angle along the particles' orbit. Some differences even for $\Lambda=0$ due to the inclusion of finite aspect ratio effects, the Shafranov shift, the elongation $\kappa \approx 1.2$ and the variation of the background parameters (like $q$ ) along the unperturbed particle orbits are found. As predicted in reference [13], the geometrical effects reduce the radial drift velocity (in this case about $12 \%$, a $1 / \kappa$-dependence would predict 20\%). The differences become even larger at the plasma edge. Also the trapped 

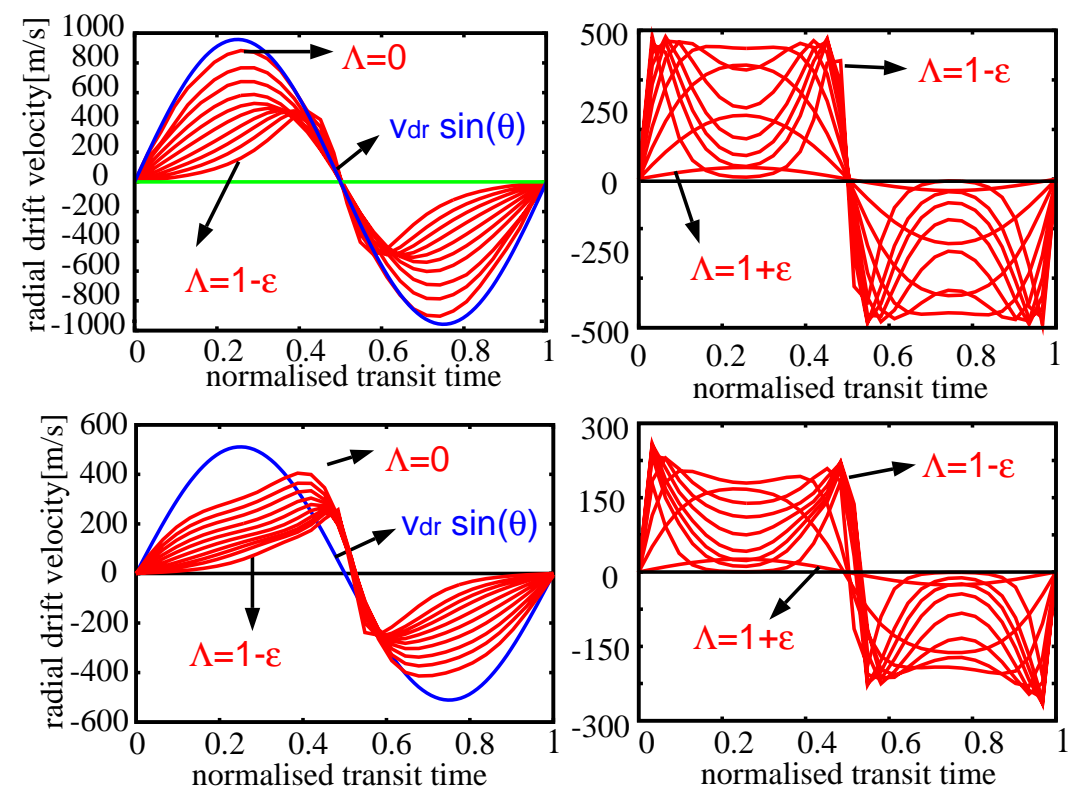

Figure 2. Radial drift velocity along one transit for circulating (left column) and trapped (right column) in the plasma center $\left(\varrho_{\text {pol }} \approx 0.37\right.$, first row) and at the plasma edge ( $\varrho_{\text {pol }} \approx 0.95$, second row). In black, the analytical approximation $v_{d r} \sin (\theta)$, in red HAGIS results. The particle energy is $2 \mathrm{keV}$. Here, $\epsilon$ is the local aspect ratio $r / R_{0} \approx 0.11$, and $\Lambda=1-\epsilon$ is an approximation for the trapped-passing boundary.

particle case is shown in fig 2 .

The next step in the analytical derivation is the integration of the propagator along the particle orbits

$$
a_{k, m, \sigma}^{G}=\frac{1}{\tau_{t} \omega} \int_{0}^{\tau_{t}} d t\left[\omega_{d}^{\theta} \cos (\theta)+\frac{\omega_{d}^{r}}{i} \sin (\theta)+\omega_{\text {prec }}\right] e^{i \sigma k \omega_{t} t}=\frac{\delta_{k, \pm 1}}{2 \omega} \omega_{d}^{\mp}+\delta_{k} \omega_{\text {prec }}
$$

and the flux surface averages

$$
\begin{aligned}
K_{m, p, k, \sigma}^{G} & =\frac{1}{2 \pi} \int_{-\pi}^{\pi} d \theta\left(\omega_{d}^{\theta} \cos (\theta)+\frac{\omega_{d}^{r}}{i} \sin (\theta)+\omega_{\text {prec }}\right) \cdot e^{-i(-p+\sigma(m-k)) \theta} \\
& =\delta_{p, m-k \pm 1} \frac{\omega_{d}^{ \pm}}{2 \omega}+\delta_{p, m \pm k} \omega_{\text {prec }}
\end{aligned}
$$

Here, $m$ is the poloidal mode number, $k$ counts the harmonics of the circulating motion, $\sigma=1$ for co-passing and -1 for counter-passing particles, $\omega_{d}^{\theta}$ is the poloidal drift and $\omega_{\text {prec }}$ the precessional drift which is small for background ions and is therefore neglected in the following analytical treatment. $\omega_{d}^{ \pm}=\omega_{d}^{\theta} \pm \omega_{d}^{r}$ and $p$ counts the harmonics of the projection operator $e^{i p \theta}$. Fig. 3 shows the radial part of the coefficient $a_{1,4,1}^{G} \cdot K_{4,4,1,1}^{G}$, normalised to the analytical result (eqn 2). A strong dependence on the pitch angle parameter $\Lambda$ can be seen, especially close to the trapped-passing boundary. Again, due to geometrical effects, there are differences $(\approx 10 \%)$ even at $\Lambda=0$.

Now the integration over velocity space is carried out. Taking into account the $k \pm 1$ resonances and summing up over co and counter-passing particles results in the factors 


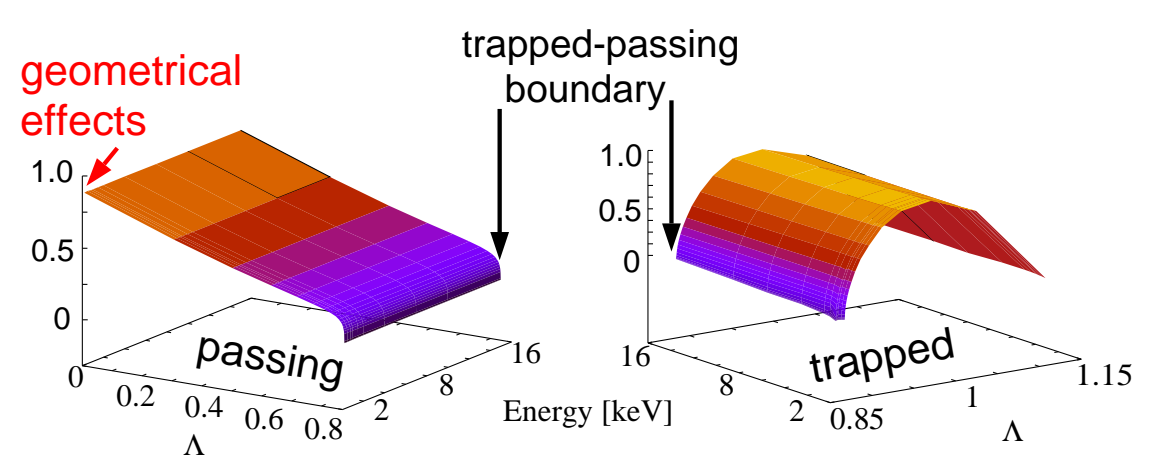

Figure 3. weighted, normalised propagator integrals as a function of pitch angle variable $\Lambda$ and energy (formula $2 *$ formula 3 ): in the fast circulating approximation, this orbit integral is 1 . The numerical result shows significant differences, even in the $\Lambda=0$ limit due to the geometrical effects.

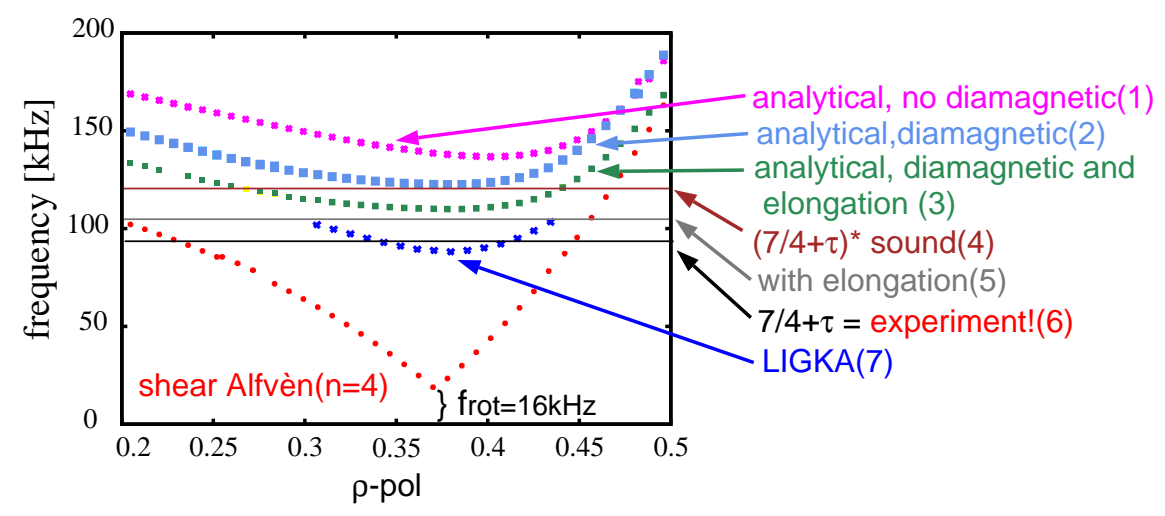

Figure 4. BAE continuum near the $q=1$ surface: down-shift of the minimum due to diamagnetic effects, elongation and particles with $\Lambda \neq 0$.

$H$ (geodesic curvature), $N$ (coupling terms) and $D$ (sound wave) as defined in eqn (1). Comparing the results is rather involved, since these coefficients depend on a guess of $\omega$. At high frequencies $\omega \gg \omega_{t}$ the agreement of the analytical and the numerical results is rather good, since only fast circulating particles contribute (here: $\omega \approx 4 \omega_{t}$ ). For lower $\omega$ also particles with $\Lambda \neq 0$ become increasingly important in the resonance integrals and therefore quite large differences can appear. For even lower frequencies, i.e. if the mode frequency is comparable to the bounce frequency $\omega \approx \omega_{b}$ the dispersion relation (1) will change significantly as also shown analytically in reference [19].

In order to demonstrate the influence of $\omega_{*}$, the geometrical effects and the applied approximations, the local solutions of the dispersion relation for the parameters of discharge \#20488 at $1.92 s$ near the $q=1$ surface are discussed. For the solving the analytical formula, a post-processing Nyquist-solver for eqn. 1 based on experimental profiles $(q, B, n, T, \nabla n, \nabla T)$ as given by the equilibrium reconstruction code CLISTE [25] is employed. Also the LIGKA code uses realistic profiles, however, in this case all integrals are solved numerically [2]. The results are plotted in figure 4. Solutions (1), (2) and (3) are based on the analytical dispersion relation, where (1) is without diamagnetic 


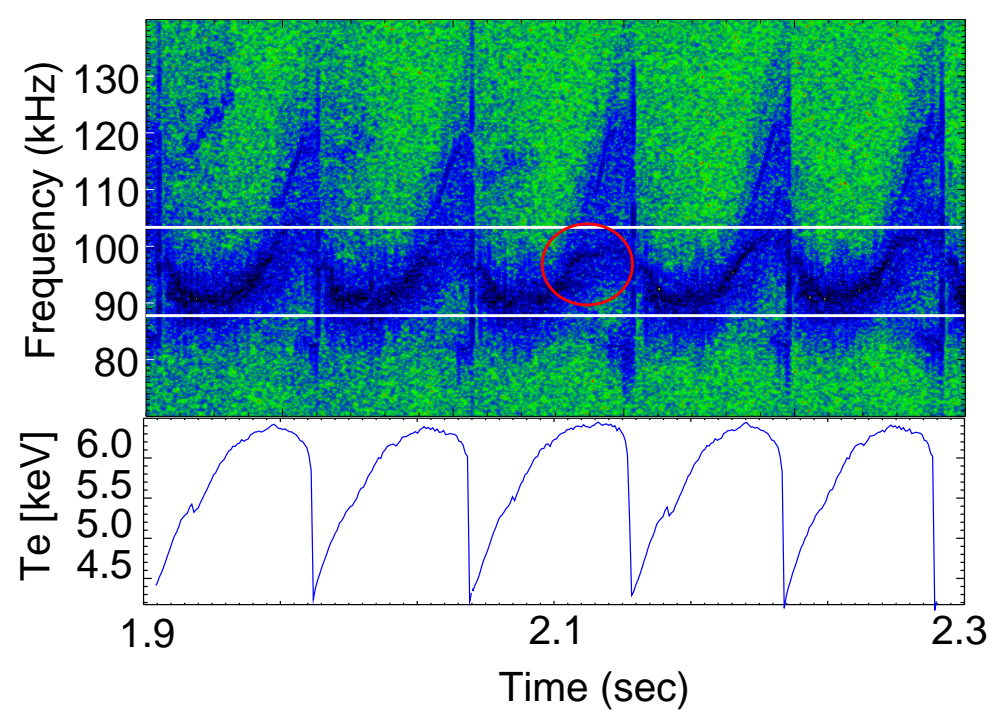

Figure 5. spectrogram (Mirnov coils) and central electron temperature evolution for \#20488

effects, (2) with diamagnetic effects and (3) is solution (2) scaled with an elongation factor of $1 / \sqrt{\kappa^{2}+1}$ [13] with $\kappa=1$.2. Solutions (4) to (6) are iterated solutions, strictly valid only for large $q$. However, since $\omega_{t} / \omega=v t h_{i} /(q R \omega) \approx 0.65$ is not too large, the iterated solutions are of some interest for the BAE regime and therefore included in figure 4. Solution (4) refers to formula (19) in reference [26], (5) represents formula (10) in ref. [13] and (6) is the simple $7 / 4+\tau$ approximation that can be derived from formula (1) in the cold ion, hot electron limit neglecting sound wave coupling and diamagnetic effects. (7) is the numerical LIGKA result. By chance, the simplest solution lines up perfectly with the experimental mode frequency, $94 \mathrm{kHz}$. Only the results containing diamagnetic effects together with an elongation correction $(3,7)$ are close to the experimental value. This leads to the conclusion that the down-shift due to these effects is crucial for understanding the experimentally measured mode frequency. Furthermore, avoiding the fast-circulating approximation and taking into account trapped particles (LIGKA-solution, 7) gives an additional significant down-shift of about 20\%. The functional dependence of this shift - obviously on the aspect ratio - will be investigated elsewhere.

\section{The BAE mode during a sawtooth cycle}

In the following, the evolution of the mode frequency (\#20488) during a sawtooth cycle will be analysed. We start with assuming a flat $q$-profile and a rather flat temperature profile $(t=1.92 s)$, see fig. 1 . According to observations at ASDEX Upgrade based on MSE measurements [27] the $q=1$ surface remains at the same radial location (here $\left.\varrho_{p o l}=0.37\right)$. Moreover, $\nabla T_{e} / T_{e}$ rises as described in section 2. The BAE continuum accumulation point at the $q=1$ surface according to formula 1 is plotted in fig. 6 . (All 


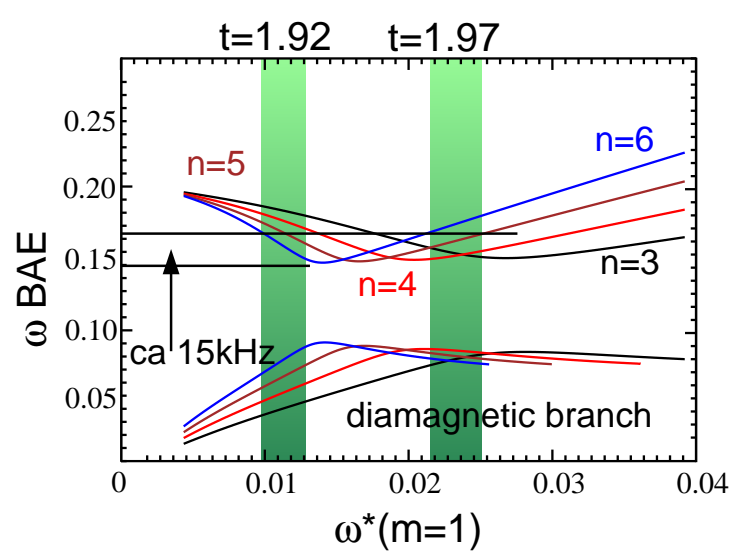

Figure 6. Analytical results (formula 1) for the BAE continuum accumulation point at the $q=1$ surface. The horizontal lines indicate the experimentally seen frequency dip of about $15 \mathrm{kHz}$ (see fig. 5). Taking into account the error bars (as indicated by the vertical columns) shows that the frequency minimum is indeed consistent with the experimental data.

frequencies are normalised to the on-axis Alfvén frequency.) The BAE-frequency passes through a minimum for $n \geq 4$. This is consistent with the experiment (see fig. 5) since the mode number analysis yields a mixture of $n=4,5,6$ for the signal between 85 and $105 \mathrm{kHz}$. The fact that modes with different toroidal mode numbers overlay is supported by shot \#22325 where a clear splitting of several toroidal mode branches caused by the Doppler shift due to NBI-induced plasma rotation was found [28]. Although keeping in mind that there is an additional downshift as discussed in the previous section, the theoretically found dip of about $15 \mathrm{kHz}$ during the sawtooth cycle (fig. 6) is consistent with the experiment where the most prominent mode is $n=5$. A rough error estimation is given as well in fig. 6 .

It should be noted that the frequency-minima are slightly different for different mode numbers $(\approx 5 \mathrm{kHz})$. In this particular discharge this splitting seems to be just compensated by the toroidal rotation $(\approx 4 \mathrm{kHz})$ that shifts the modes - more or less on top of each other.

The strong frequency-chirp just before the crash cannot be explained by diamagnetic effects, since the temperature has already reached its maximum $20-30 \mathrm{~ms}$ before the crash (see fig. 5). Since a part of the mode follows exactly the temperature evolution (mode stops chirping $20 \mathrm{~ms}$ before the crash, red circle in fig. 5 ) one can conclude that these modes are different in nature. This will be proven in the following.

Unfortunately, for discharge \#20488 no radial information about the mode signal is available. However, in the relatively similar shot \#23824 (800kA, 4.5MW ICRF, $B_{0}=2 T$, profiles given in fig. 1 and 8) the upgraded fast (2MHz sampling rate) SXR diagnostics allow to get a clear picture of the radial mode localisation. Fig. 7 demonstrates, that the signal consists of chirping modes, localised inside the $\varrho_{\text {pol }} \approx 0.25$ (central SXR channels) and another part that is inside the $\varrho_{\text {pol }} \approx 0.4$ surface that 

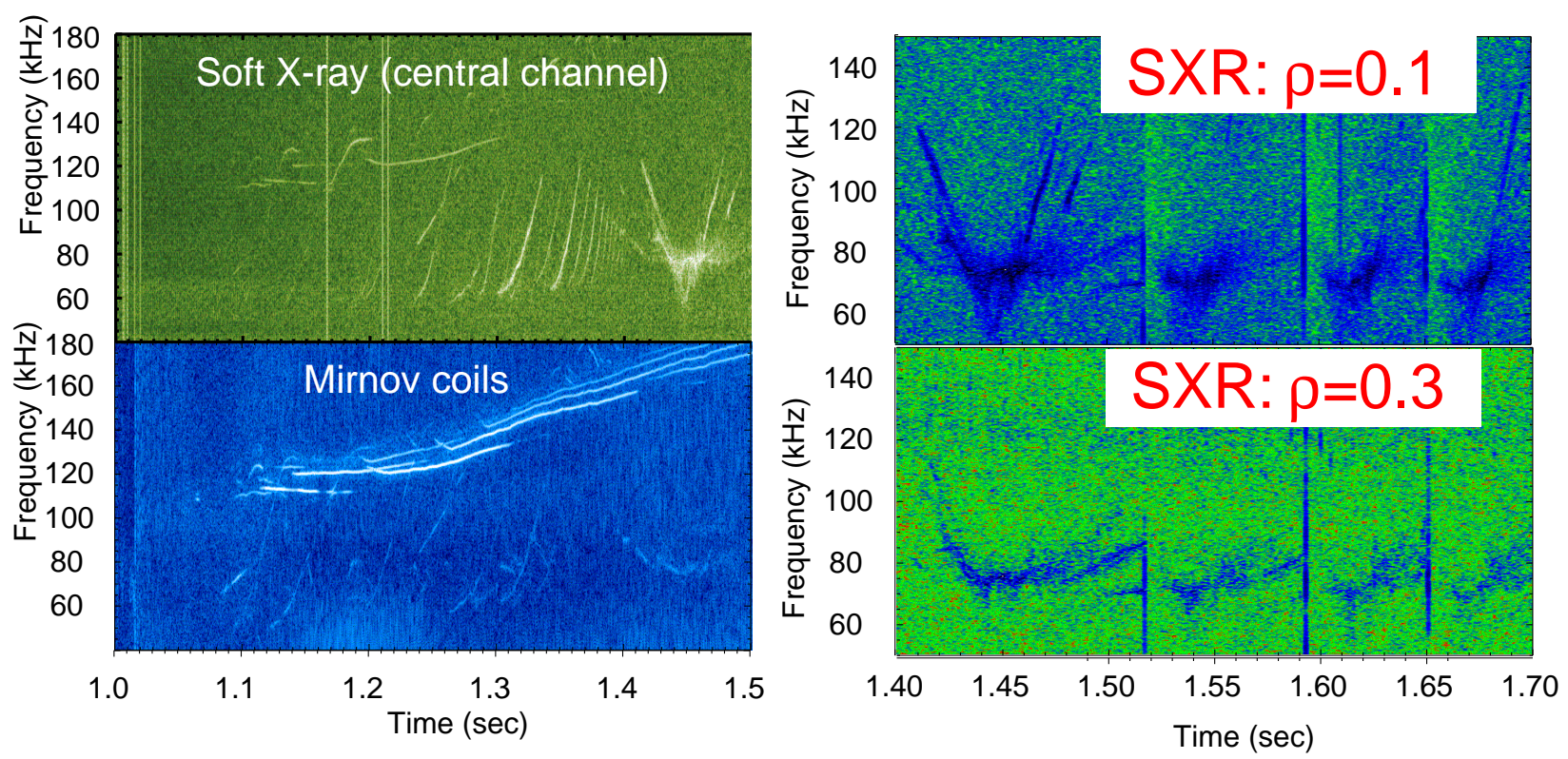

Figure 7. Soft-X-ray and magnetic spectrograms for \#23824

exhibits very similar features as the BAE mode described above. As can be seen in the SXR cameras (fig.7), the appearance of Alfvén cascades (or reversed shear Alfven eigenmodes, RSAEs) indicates that the q-profile is reversed due to the current diffusion process in the beginning of the discharge.

Here, the phase before the first sawtooth crash at $\sim 1.5 \mathrm{~s}$ is investigated more closely. At $t=1.45 \mathrm{~s}, q_{\min }$ is dropping below 1 , causing the RSAE mode to change from chirping-down to chirping-up (as indicated by the arrows in the $n=4$ kinetic spectrum of fig. 8). Since the $q_{\text {min }}$ surface moves inwards, the chirping-up after $1.45 \mathrm{~s}$ is only seen in the very central channels. (A possible theoretical interpretation of the chirping down RSAE has been proposed in ref. [29], a numerical investigation with LIGKA has been started). At the same time also the minimum in the kinetic Alfvén continuum due to the geodesic curvature starts to form (fig. 8 ). Therefore, a BAE mode with a lower toroidal mode number becomes visible at slightly higher frequency (smaller $\omega_{*}$ downshift). For this shot, a definitive mode number identification was not possible, however from an estimation of $d q_{\min }(t) / d t$ (by identifying the mode numbers of the RSAEs and TAEs between $1.1 s$ and $1.4 s$ and assuming a constant current profile relaxation) a mode number of $n=4$ is most likely.

Since the cascade modes can be also seen at later times, e.g. just before the crash at $t=1.7 \mathrm{~s}$ it can be concluded that there is some shear reversal (or at least a very flat q-profile) during the sawtooth cycles as reported recently by Alcator C-Mod [30]. At $t=1.7 \mathrm{~s}$ one can determine $q_{0}$ : extrapolating the chirping mode to $t=1.71 \mathrm{~s}$ resulting in $\sim 150 \mathrm{kHz}$ as the maximum mode frequency and assuming again $n=4$ leads to a $q_{0}$ of 0.96 . This value is in agreement with q-profile reconstruction studies recently carried out at ASDEX Upgrade [31]. 

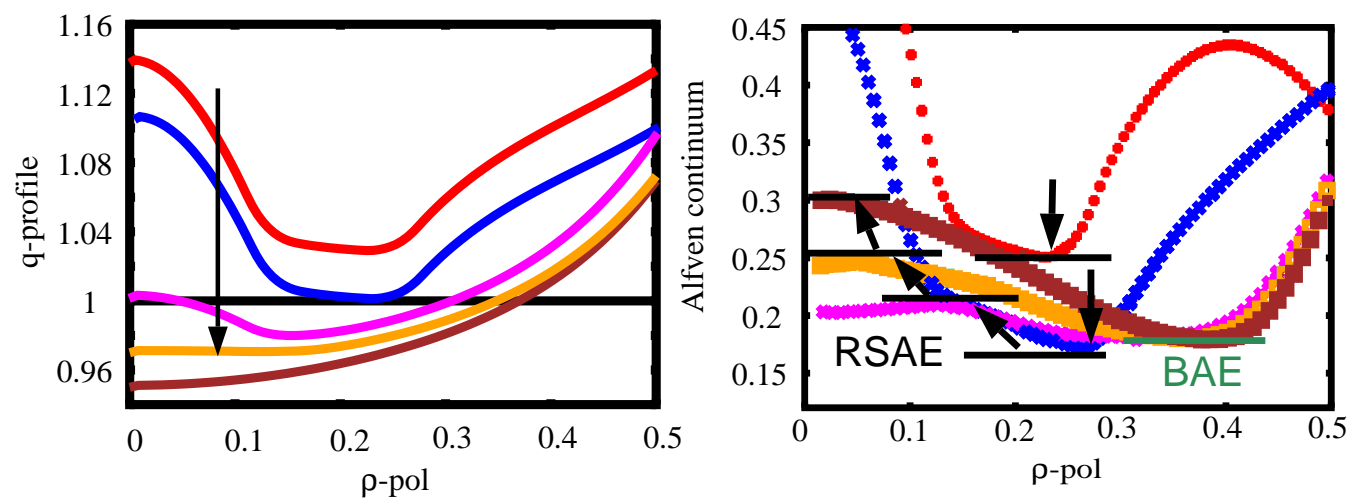

Figure 8. A series of manually modeled q-profiles (by changing the input equilibrium current profile) qualitatively consistent with the current diffusion process during the current ramp-up phase. The different colors refer to different time points (from top to bottom): red: $1.41 \mathrm{~s}$, black: $1.45 \mathrm{~s}$, pink: $1.47 \mathrm{~s}$, yellow:1.49, brown 1.51 . On the right, the corresponding kinetic continua. The arrows and the black bars indicate the frequency behaviour and the localisation of the RSAE mode, the green bar (above the label BAE) indicates the formation of the BAE gap at $\varrho_{\text {pol }}=0.37$.

\section{Conclusions and Outlook}

In order to understand the properties of core-localised modes at ASDEX Upgrade, a detailed kinetic analysis employing various analytical and numerical models has been carried out. It was found that several downshift mechanisms of the BAE/GAM continuum accumulation point play a role: diamagnetic effects, elongation and the correct treatment of finite $\Lambda$ trapped particles. The latter effects were found by rederiving the well-known dispersion relation within the LIGKA model and carrying out a step-by-step comparison with the analytical results. This discussion stresses the importance of a fully consistent kinetic treatment of modes in the BAE and BAAE range. It also shows that a simple renormalisation of fluid/MHD theory results - as suggested in ref [32] - is not sufficient due to the non-linear behaviour of the dispersion relation and the trapped particle dynamics for low frequencies $\sim 2 \omega_{t}>\omega>\omega_{b}$.

Comparison with experimental data leads to the conclusion that based on the discharges discussed above, there seems to be no evidence that contradicts the interpretation of the experimentally found mode [20] as a BAE mode. The next step will be to determine the fast particle contribution (resonant and non-resonant part [33]), discuss the mode existence criteria and the energetic-particle branch of these modes. Also non-linear calculations employing an extended version of the HAGIS code are on the way, explaining in detail the loss mechanisms due to these modes.

Finally, quantitative conclusions for the sawtooth physics can be drawn from an accurate interpretation of core localised modes. In particluar, if BAEs and RSAEs are present simultaneously, two independent constraints for the q-profile evolution can be given. 
The authors would like to acknowledge with gratitude H.L. Berk and F. Zonca for elucidating discussions on the dispersion relations, L. Urso for the help with experimental ASDEX Upgrade data and the diagnostic teams at ASDEX Upgrade for their support.

\section{References}

[1] F. Zonca et al, Plasma Phys. Control. Fusion 38, 2011 (1996)

[2] Ph. Lauber et al, J. Comp. Phys., 226/1 (2007)

[3] A.D. Turnbull et al Phys. Fluids B 5, 2546 (1993)

[4] B. Heidbrink et al, Phys. Rev. Letters 71 (1993)

[5] N. Winsor et al Phys. Fluids 11, 2448 (1968)

[6] F. Zonca, S. Briguglio, L. Chen, G. Fogaccia, T.S. Hahm, A.V. Milovanov and G. Vlad, I1.001. Physics of Burning Plasmas in Toroidal Magnetic Confinement Devices. Presented at the 33rd EPS Conference on Plasma Physics, 19-23 June 2006, Rome, Italy; Plasma Physics and Controlled Fusion 48, B15-B28, (2006)

[7] X. Garbet et al, Theory of Fusion Plasmas (AIP), Vol. CP871, p. 342 (2006)

[8] F. Zonca and L. Chen, EPL 83, 35001 (2008)

[9] C. Nguyen et al, Phys. Plasmas 15, 112502 (2008)

[10] A. Smolyakov et al, Plasma Phys. Controlled Fusion 50, 11 (2008)

[11] F. Zonca et al, Phys. Plasmas 6, 1917 (1999)

[12] A. Elfimov et al, Phys. Plasmas 16, 034501 (2009)

[13] Z. Gao et al, Phys. Plasmas 15, 074502 (2008)

[14] P.Villard et al, AIP Conf. Proc. 871, 424 (2006)

[15] Ph. Lauber et al, 35th EPS conference on Plasma Phys., ECA 32 (2008) O-4.030

[16] H. Qin et al, Phys. Plasmas 5, 1035 (1998)

[17] F. Zonca, private communication (2008)

[18] F. Zonca et al, Nucl. Fusion 49085009 (2009)

[19] F. Zonca and I. Chavdarovski, Paper P1.133. Presented at the 36th EPS Conference on Plasma Physics and Controlled Fusion, Sofia, Bulgaria, June 29 - July 3, (2009)

[20] M. Garcia-Munoz, et al, Phys. Rev. Letters 100, 055005 (2008)

[21] V. Igochine et al, Nucl. Fusion 43, 1801 (2003)

[22] F. Ryter et al, Phys. Rev. Letters 862325 (2001)

[23] J. Stober et al, Nucl. Fusion 431265 (2003)

[24] Pinches S.D. et al, C. Ph. Comm., 111 (1998)

[25] P. Mc Carthy, Phys. Plasmas 6, 3554 (1999)

[26] H. Sugama, T.-H. Watanabe,Phys. Plasmas 13, 012501 (2006)

[27] J. Hobirk, private communications, (2009)

[28] A. Elfimov et al, Paper P1.141. 'Rotation effect on geodesic ion Alfvén modes in hot tokamak plasmas', Presented at the 36th EPS conference on Plasma Physics, Sofia, Bulgaria, June 29 July 3, (2009)

[29] N. Gorelenkov, Phys. Plasmas 15, 110701-1 (2008)

[30] E. Edlund et al, Phys. Rev. Lett. 102, 165003 (2009)

[31] K. Sassenberg et al, Plasma Phys. Control. Fusion 51065003 (2009)

[32] N. Gorelenkov et al, Phys. Plasmas 16, 056107 (2009)

[33] F. Zonca et al, Plasma Phys. Control. Fusion 48, 537 (2006) 